\title{
Redescubriendo los caminos antiguos desde Colombia
}

La Colombie: un point de départ pour la redécouverte des vieux tracés routiers Re-discovering the ancient ways from Colombia

\section{Sofía Botero Páez}

\section{(2) OpenEdition}

\section{Journals}

Edición electrónica

URL: https://journals.openedition.org/bifea/3505

DOI: $10.4000 /$ bifea.3505

ISSN: 2076-5827

Editor

Institut Français d'Études Andines

Edición impresa

Fecha de publicación: 1 diciembre 2007

Paginación: 343-352

ISSN: 0303-7495

\section{Referencia electrónica}

Sofía Botero Páez, «Redescubriendo los caminos antiguos desde Colombia», Bulletin de l'Institut français d'études andines [En línea], 36 (3) | 2007, Publicado el 01 junio 2008, consultado el 07 diciembre 2022. URL: http://journals.openedition.org/bifea/3505 ; DOI: https://doi.org/10.4000/bifea. 3505

\section{cc) (i) $\Theta$}

Creative Commons - Atribución-NoComercial-SinDerivadas 4.0 Internacional - CC BY-NC-ND 4.0 https://creativecommons.org/licenses/by-nc-nd/4.0/ 


\title{
Redescubriendo los caminos antiguos desde Colombia
}

\author{
Sofía Botero Páez*
}

\begin{abstract}
Resumen
El artículo sintetiza y ordena información relacionada con los caminos en América y ofrece elementos útiles para la definición de un objeto de estudio a partir del cual es posible acercarse al entendimiento de fenómenos culturales, físicos y naturales pero también de procesos históricos, sociales y económicos de sus constructores y transeúntes.
\end{abstract}

Palabras clave: América, Colombia, caminos, tecnología, arqueología, historia de indígenas

\section{La Colombie : un point de départ pour la redécouverte des vieux tracés routiers}

\section{Résumé}

L'article propose une synthèse raisonnée de l'information dont on dispose sur les routes américaines. II offre des éléments permettant de circonscrire un objet d'étude à partir duquel on pourra saisir l'explication de phénomènes culturels, physiques et naturels et comprendre les processus historiques, sociaux et économiques dans lesquels étaient engagés les constructeurs de ces chemins et leurs utilisateurs.

Mots clés : Amérique, Colombie, chemins, technologie, archéologie, histoire des indigènes 


\title{
Re-discovering the ancient ways from Colombia
}

\begin{abstract}
The article synthesizes and orders information related to the ancient roads in America and offers useful guidelines for their definition. In this manner we can reach an understanding not only of their cultural, physical and natural aspects but also of the historical, social and economic processes involved.
\end{abstract}

Key words: America, Colombia, trails, technology, archaeology, indigenous history

\section{INTRODUCCIÓN 1}

«[...] Desde la provincia de Arma hasta la de Çenufana habrá xx leguas y desde Çenufana a Aburrá puede haber seis, en todo este camino hay grandes asientos de pueblos antiguos e muy grandes edificios de caminos hechos a mano e grandes por los sierras e medias laderas que en el Cuzco no los hay mayores [...]» (Robledo, 1993 [1539-1542]: 350).

Este texto se escribe desde la arqueología y la historia que se conoce y se ha desarrollado en Colombia, y pretende fundamentalmente llamar la atención sobre la importancia de su red vial prehispánica, sintetizar una gran cantidad de información relacionada con los caminos y proponer un objeto de estudio, a partir del cual sea posible acercarse al entendimiento de múltiples aspectos de la vida de sus constructores y de los entornos geográficos por los que circularon.

El interés del estudio de los caminos tiene múltiples razones: los caminos son —sin duda-, los ejes físicos a través de los cuales se concretan los procesos de poblamiento y se articulan las relaciones sociales y económicas que finalmente consolidan una determinada región o sociedad. La dificultad que conlleva su construcción y destrucción, los convierte en vestigios persistentes, posibles de identificar y rastrear junto con la historia de sus transeúntes. La complejidad técnica que implica su trazado y construcción, contiene un inmenso potencial para informar sobre formas y procesos que implican muy diversos conocimientos. Al incorporar a su análisis la geografía y las características físicas de los territorios que atraviesan, su estudio hace posible concretar, de la manera más completa, los modelos y propuestas derivadas de las nuevas corrientes del análisis arqueológico denominadas como «arqueología del paisaje».

Al hablar de caminos podemos estar hablando de huellas o «rastros» en el paisaje, que apenas son perceptibles a los conocedores, o que por el contrario se visualizan en sólidas estructuras pavimentadas como en la actualidad; apisonadas, afirmadas, como las de los romanos; o empedradas como las de los incas en el Perú, o los tairona y los hevexico en Colombia, solo para mencionar algunos ejemplos. Finalmente, un elemento que cobra relevancia al momento de definir la importancia de los caminos, es que ellos son el símbolo del avance de la cultura frente a la naturaleza, los caminos aparecen como los agentes de la civilización, a través de ellos llega el progreso, se transforma el paisaje y con su abandono también llega la decadencia.

1 La investigación que permite presentar este texto fue financiada por el Comité para el Desarrollo de la Investigación (CODI) de la Universidad de Antioquia, a través del Centro de Investigaciones de la Facultad de Ciencias Sociales y Humanas - $\mathrm{CISH}-$. 


\section{CONTEXTO CONCEPTUAL}

Una de las dificultades que debe afrontar este ejercicio es la permanente referencia a vías y caminos. Éstos son mencionados de una u otra manera en prácticamente todos los textos de historia y arqueología; sin embargo, a pesar de la reiteración, resulta claro que el concepto mismo de camino, vía de comunicación, ruta, trocha o sendero, toma muy distintas connotaciones, no solo en cada uno de los autores sino a lo largo de los mismos textos; en general éstas palabras se utilizan como sinónimos — cuyo uso además es valorativo y no descriptivo- - y prácticamente en ningún caso, se realiza una definición explícita de ellas.

A riesgo de realizar una generalización abusiva y excluyente, nos atrevemos a proponer que hasta el momento el análisis de los caminos se ha hecho aplicando modelos económicos derivados del capitalismo, privilegiando el análisis sobre el tipo de mercancías, y que asumen como determinantes de su existencia la necesidad de maximizar los beneficios económicos y reducir los costos tanto en el transporte como en su construcción; se da por sabido que su construcción implica una alta organización y distribución de recursos sin que se haya explorado sistemáticamente en la forma, direccionalidad y racionalidad de las vías mencionadas. Así mismo, la mayoría de veces se asumen como obvias las relaciones que establecen los transeúntes de estas vías, y en general se ignora el conjunto total de las redes jerárquicas — físicas y sociales_ que establecen sus transeúntes y moradores.

De otro lado, el estado actual del estudio sobre los caminos está sin duda marcado por la dificultad de establecer cronologías precisas y absolutas asociadas a ellos; la enorme variabilidad y dinámica de utilización que están asociadas a las rutas y vías de comunicación, sumadas a la pervivencia que tienen las técnicas y los materiales constructivos utilizados, ha permitido a los distintos pobladores reconocerlos y asumirlos como suyos, planteando a los investigadores dificultades de orden técnico y epistemológico que no han sido explícitamente reconocidos ni afrontados en ninguna de las etapas de la investigación, ni de la reflexión.

Hay que resaltar la notoria escasez de trabajos que se ocupan de la misma existencia física de los caminos, incluso cuando se trata de la descripción de caminos específicos; excepcionalmente se alude al trazado, a las distancias, variantes, o necesidades de mantenimiento. El estudio de la red vial incaica es tan excepcional, que justamente lo podemos considerar como la excepción que confirma la regla (Hagen, 1977; Hyslop, 1992).

Finalmente, consideramos necesario reiterar que las ideas que subyacen en las afirmaciones y estudios hasta el momento abordados, al no hacer explícitos sus referentes de comparación, asumen como ideal económico necesario la expansión e intensificación de un comercio mercantilista con España, de donde se importan productos de lujo que se consideran signos de progreso, desarrollo y civilidad; ignoran o al menos se invisibilizan, la presencia y dinámica social, política y comercial de aquellos que por una u otra razón no tuvieron acceso directo a las instituciones de poder o a las redes de comercio oficialmente reconocidas y, sobre todo, se borran los vestigios de la historia y presencia indígena en extensos territorios de la región.

Desde la arqueología, un elemento que nos ayuda a explicar la situación, está sin duda en el marco conceptual y geográfico más amplio en el que se ha desarrollado la disciplina en esta parte del continente. Desde un punto de vista evolucionista, y utilizando como marcadores territoriales espectaculares vestigios de templos y ciudades, los arqueólogos, corrientemente se refieren a la existencia de una «América Nuclear», terminología con 
la que se reconoce la importancia como polos de desarrollo de dos «grandes áreas de civilización aborigen»: Mesoamérica, incluidos los mayas y los aztecas, y los Andes en donde se asentaron los incas. Para solucionar las dificultades teóricas que genera la lejanía entre estos dos centros, a lo que está en medio, se la ha definido como «área intermedia», en donde se asume que:

«nunca surgieron grandes imperios, ni hubo extensas ciudades, ni palacios, ni fortalezas, ni templos monumentales, como en Mesoamérica y Andes Centrales» (Reichel-Dolmatoff, 1997 [1986]: 3).

Estas ideas no han sido sistemáticamente puestas en cuestión y al contrario gozan de gran popularidad tal y como puede corroborarlo cualquier persona que tenga acceso a internet. El «área intermedia» así definida, abarca un extenso territorio en el que se incluye países que hoy reconocemos como Costa Rica, Panamá, Nicaragua, Honduras, El Salvador, Colombia, Venezuela y Ecuador, también Cuba, las Antillas, y algunas islas en el Pacífico. A pesar de la abigarrada diversidad geográfica y social de este inmenso territorio, su historia es prácticamente desconocida (figs. 1-2).

En Colombia, importantes y recientes publicaciones sobre caminos plantean el interés y alcance que tiene su estudio (Salgado López, 1986; Oyuela Caicedo, 1990; Patiño, 1991; Arciniegas, 1995; Velandia, 1996; Cardale de Schrimpff, 1996; Vélez \& Botero, 1997; Vélez Escobar, 1999; Cardale de Schrimpff \& Herrera, 1995; 2000; Languebaek et al., 2000; González Escobar, 2001; Jiménez et al., 2005), sin embargo se ha aceptado, sin revisión ni escrutinio la idea de que los indígenas no desarrollaron un complejo vial importante, e incluso no necesitaron caminos, dado su precario estado de desarrollo.

Al hablar de sus caminos ha sido un lugar común, aceptado y repetido por historiadores y académicos, considerar la región como aislada y periférica, por carecer de caminos importantes o adecuados para su desarrollo; la comparación se hace con relación a centros de importancia política y económica como Teotihuacan o Cuzco; se ha considerado que las dificultades que impone la arrugada topografía en gran parte de su territorio, hizo extremadamente complicada su construcción, a la vez que explica el pésimo estado de los pocos caminos existentes (Patiño,

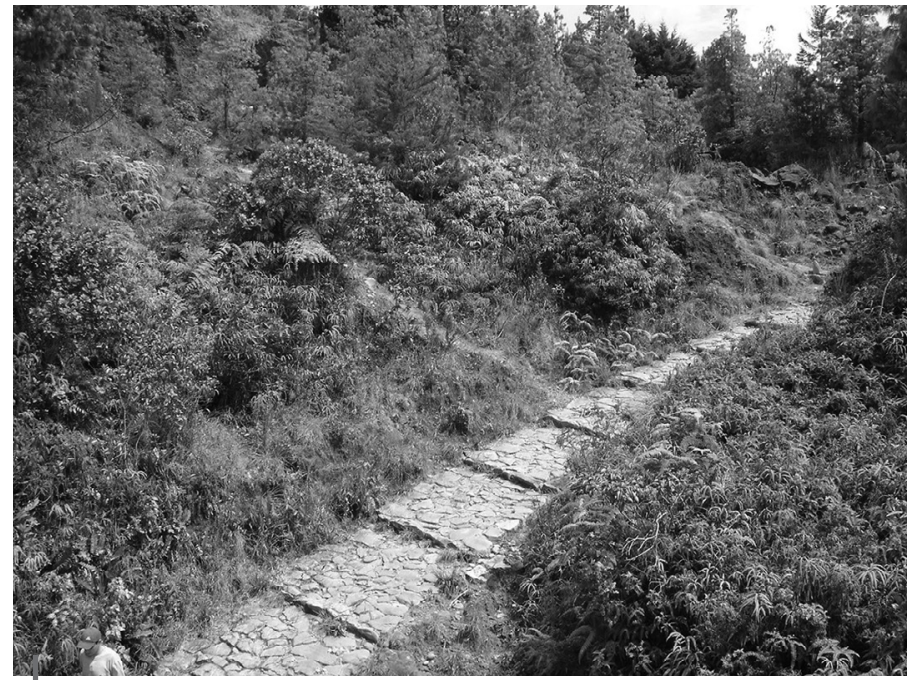

Figura 1 - Tramo del camino reconocido por el cronista Cieza de León como «un gran camino que va al Oriente»
1991: 25-26; Langebaek, 1995; 2001).

En concordancia con estas ideas, se promulga que fueron los españoles quienes construyeron los caminos «reales» aún existentes, y que comenzaron a hacerlo desde el inicio mismo de la conquista; es interesante resaltar que se asume que aquí, en Colombia, los españoles usaron las técnicas romanas en la construcción de los caminos por los que transitaron los primeros conquistadores:

«Las técnicas de construcción de calzada que encontramos en los caminos reales 
construidos a lo largo de Colombia tienen un origen remoto en el Imperio Romano y el desarrollo medieval de los caminos en España [...] Los romanos llegaron a distintos tipos de calzadas, las cuales simplificadas, se continuaron ofreciendo en la Edad Media Ibérica» (Langebaek et al., 2000: 72).

Cuando se reconoce que los indígenas tenían caminos, el esfuerzo que se hace es para demostrar que eran «cortos» o de poca importancia:

«[...] las comunicaciones entre las diferentes subregiones del país tenían poca importancia en la formación de redes amplias de intercambio económico. Quiero enfatizar aquí el caso de los muiscas y su supuesta relación con grupos muy alejados. Al igual que lo planteado por Earle (1987a) con respecto a sociedades complejas del Perú y del Pacífico, en Colombia prehispánica fuertes

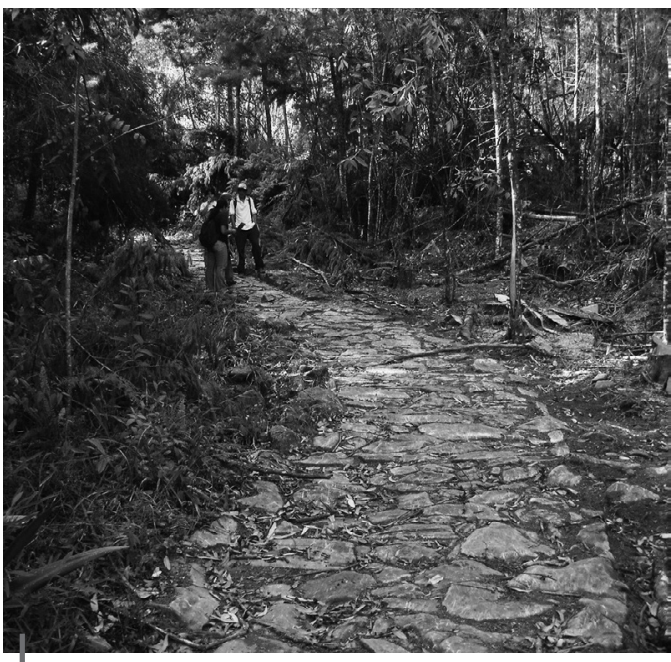

Figura 2 - Camino sobre ladera oriental del Valle de Aburra (zona rural de la ciudad de Medellín) diferencias de medio ambiente resultaron en contrastes en sistemas productivos, no en el desarrollo de simbiosis entre regiones muy apartadas. Los cacicazgos colombianos tendieron a ser unidades de producción de comida autosuficientes. Eventualmente se puede documentar que el intercambio de materias primas por objetos elaborados alcanzó cierta importancia en algunas regiones del país [...] sin embargo aún en este caso, el tamaño de los circuitos de intercambio fue más reducido de lo que usualmente se piensa [...]» (Langebaek, 1995: 35).

Así las cosas, lo que es innegable es que no se han dedicado esfuerzos constantes y sistemáticos a abordar y menos pensar «eso» que pudiera haber sido la red vial prehispánica, aunque la evidencia documental y arqueológica señala una amplia circulación de bienes y mercancías, en distancias que implican la existencia física de caminos de tierra y agua,

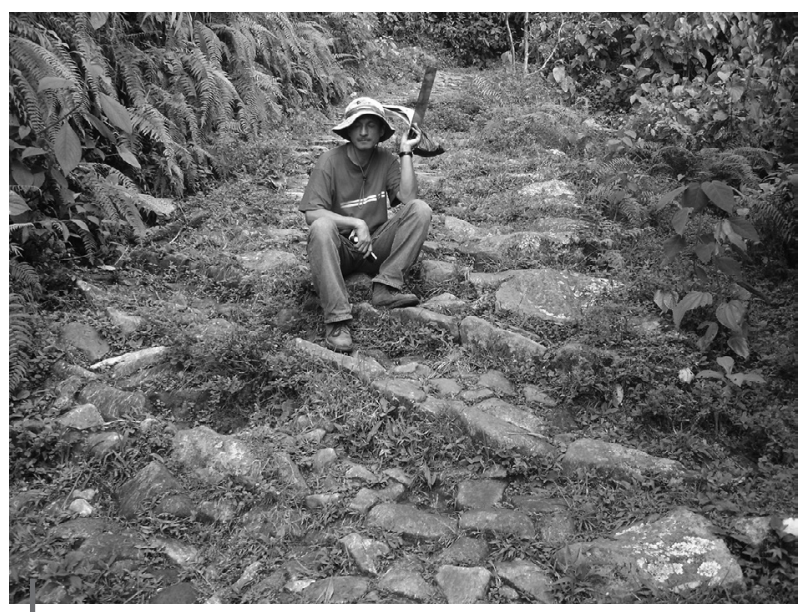

Figura 3 - Municipio de Jericó

Obsérvese la obra para el drenaje y el reconocimiento de territorios y relaciones, hasta el momento no consideradas por los estudiosos de la región (fig. 3). Entre ellos destacamos el extenso circuito (desde el extremo noroccidental de Colombia hasta llegar a la desembocadura del Orinoco), documentado por el investigador Adam Szasdi Nagy (1983), quien logra establecer las rutas de distribución del cobre y del guanín que según informaron los conquistadores y cronistas del siglo $\mathrm{XVI}$, circulaba corrientemente en las Antillas y Centroamérica, aportando elementos que corroboran la idea de que las patenas (guanines), caracuries —narigueras_ y las «aguilillas», 
localizadas en las Antillas y el Caribe, son de origen colombiano2. Los metales exportados desde los Andes colombianos alcanzaron las Antillas y bien pudieron alcanzar a los pueblos que vivían junto al Amazonas, pero ¿̇y qué pasaba hacia el sur?

Por otra parte, los investigadores de la colonia han centrado su interés en el análisis del comercio y en establecer la cantidad y tipo de productos que circulaban, temas sobre los cuales existe una innumerable cantidad de estudios y documentación, en la que se afirma insistentemente que el mal estado de los caminos hizo muy difícil, cuando no imposible, el tráfico fluido y corriente de mercancías y animales. Sin embargo, no ha sido suficientemente subrayado que fue con estos mismos argumentos que se justificó la utilización de seres humanos como animales de carga, a pesar de ser una práctica amplia y enérgicamente prohibida desde los albores de la Conquista (Patiño, 1991; Botero Páez, 2005).

Llamamos la atención sobre el hecho de que si bien los caminos incas en la actualidad gozan de gran popularidad y reconocimiento, durante la colonia también se consideraron horrorosos e intransitables; el informe que en el año de 1773, escribe un cura cuya doctrina se ubicaba en el valle del Cañete (departamento de Lima, Perú), no deja ninguna duda al respecto:

«Los caminos los más horrorosos de la provincia por lo que hace preciso transitar mucha parte de ellos a pie, por el horror que causa pasarlos en las cabalgaduras; pues para llegar en derechura esta la horrorosa baxada que consta de cinco mil y más escalones» (Archivo Arzobispal de Lima, Sección Visitas Eclesiásticas Curatos, Legajo, expediente 6, año de 1773, 6 folios, citado por Coello Rodríguez, 2000: 176).

Por lo cual nos resulta paradójico que tampoco se hayan formulado preguntas sobre los caminos construidos por los españoles. Tratándose de nuevos territorios, resultaría de gran interés precisar dónde y en qué circunstancias debieron construir o al menos transformar los caminos para permitir el paso continuado de caballos, ganado y de cientos de hombres cargados con cosas jamás vistas en esta parte del continente, pesadas y muy difíciles de transportar entre las fuertes pendientes andinas.

\section{ELEMENTOS PARA RECOMPONER EL ESTUDIO DE LOS CAMINOS}

En primer lugar es necesario destacar que en Colombia existe una muy extensa e intrincada red de caminos catalogados en la actualidad como reales, de herradura, veredales, trochas y senderos, entre los que se encuentra un sinnúmero de caminos empedrados, algunos de los cuales llamaron poderosamente la atención de los conquistadores.

Los denominados caminos «reales», por ser los de mayor tránsito y de mayor envergadura, captaron el interés tanto de la administración colonial como el de los estudiosos actuales. Invariablemente asociados al accionar español se ha asumido tacita y explícitamente que fueron trazados y construidos por los españoles; sin embargo, llama la atención que en sus descripciones y quejas, se refieran, permanentemente, a caminos empedrados, a pesar de la evidente dificultad que este tipo de vías representa para el tránsito de los equinos:

«En la época de la colonia por las estrechas escalinatas construidas con piedras, sendas que se iniciaban en el piedemonte y subían a las cumbres andinas, por esos caminos empedrados trepaban las fatigadas mulas, emitiendo por intervalos sus resoplidos, guturales sonidos, en tanto que los arrieros las azuzaban con gritos y silbos. [...] En veces los caminos eran estrechos senderos cavados en las rocas de altísimos riscos desde donde se oteaban profundos abismos o se veían muy arriba

2 Una reciente síntesis sobre este tema es posible consultarla en Sáenz Samper (2001). 
los vértices paramunos con herbazales azotados por el viento y golpeados por el granizo. [...]» (Repizo Cabrera, 1994: 86-87).

Uno de los rasgos que consideramos característicos de los caminos empedrados prehispánicos, tanto los localizados en Colombia, como los reconocidos como «incas», son los peldaños o escaleras; elemento constructivo estructural que permite el manejo de las pendientes, pero que es completamente inadecuado a las necesidades y características de mulas y caballos e impedía irremediablemente el uso de la rueda. Consideramos que esta característica no solo está adaptada a las condiciones de la quebrada topografía de la región y responde adecuadamente a la exigencias de suelos altamente deleznables, sino que manifiestan su carácter eminentemente pedestre (fig. 4).

El arqueólogo John Hyslop y su equipo de investigación, interesados en entender los aspectos técnicos que involucró la construcción de la red vial incaica (con 23189 km hasta donde se conoce), concluyen que las escalinatas tienen la ventaja de permitir una traza relativamente recta en puntos donde el terreno es muy quebrado:

«Los escalones con frecuencia son de piedras de campo ancladas en la superficie de la pendiente. Cuando se usan escalones en abundancia tienden a producir el efecto de angostar el camino, lo que nos lleva a observar que un camino de 6 metros de ancho sobre una superficie plana puede verse reducido a un ancho de 1 a 3 metros allí donde se usa una considerable cantidad de escalones ya que estos requieren un trabajo sustancial para su construcción y mantenimiento. Los cronistas tempranos de la conquista abundan en comentarios sobre estos escalones en el camino incaico. Su frecuente mención se debe sin duda a lo impresionante de su construcción (algunas cuestas tienen miles de escalones) y porque eran difíciles de transitar a caballo por el temor que les ocasionaba [...]» (Hyslop, 1992: 74-78).

Considerando la cantidad de vestigios que se conservan de los caminos empedrados, tanto en el Perú como en Colombia,

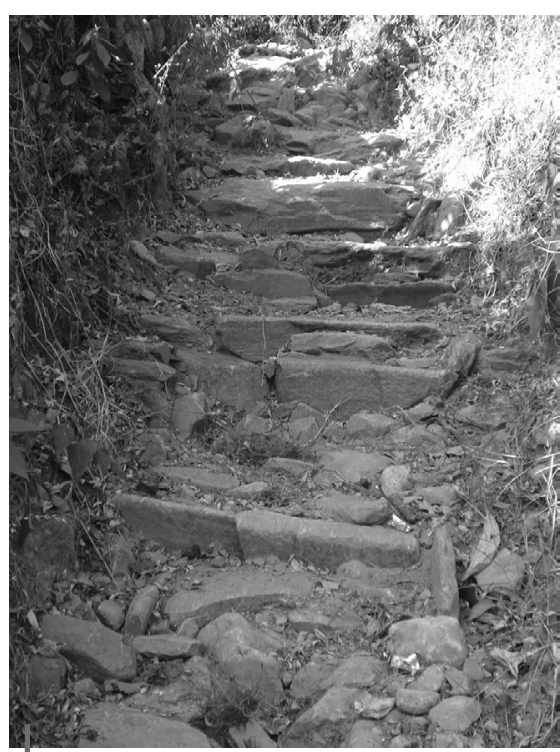

Figura 4 - Municipio de Titiribí Ancho de la banca (1 m) en lugares donde la humedad y pluviosidad es muy alta, no creemos acertada la idea de Hyslop sobre la necesidad de permanente mantenimiento en estas vías, ya que aquí, con un tráfico sustancialmente pedestre, las lajas de piedra no soportan pesos diferenciales que terminarían por removerlas, cosa que sí ocurriría en las vías con tráfico de carretas o de equinos. Si bien resulta claro que la existencia de caminos empedrados no la podemos considerar como la única ni más representativa característica de la red vial prehispánica, metodológicamente, su utilidad referencial es indudable en tanto nos proporciona elementos para emprender la caracterización y definición de nuestro objeto de estudio.

\section{ALGUNOS PUNTOS DE PARTIDA}

En tanto investigadores, ajenos a la sociedad o sociedades que suponemos construyeron los caminos, lo que pretendemos es conocer las distintas actividades que ellos posibilitaron, 
asumimos que en realidad lo que vamos a encontrar es una red de caminos, en la que se entrelazan todas las actividades y lugares en los que vivieron sus constructores. Por tanto la existencia e importancia de un camino debe referirse a la existencia e importancia de los otros, la mayor o menor importancia que se le asigne a un lugar o un camino es una noción que debe ser construida a partir de la información disponible tanto del camino como de sus constructores y transeúntes, y en ningún caso podría definirse a priori. No necesariamente un camino que pudiéramos identificar como «extenso» — por comparación con otros o porque comunica lugares que consideramos distantes - es un solo camino; es probable que sea más bien el resultado de la suma de varios, por definición más cortos, que se fueron construyendo a lo largo del tiempo o incluso simultáneamente, pero no obedeciendo a una sola lógica de necesidades o voluntades. El seguimiento del camino y el análisis de las técnicas constructivas, ayudaría a resolver este asunto. En principio, la definición de una tipología de los caminos tendría meramente propósitos metodológicos, ayudaría a entender la ubicación e interés de un camino específico dentro de la red más amplia y proporcionaría además una estrategia expositiva que permitiría ordenar de manera comparativa los datos y la argumentación.

No es solo a partir del hallazgo de «grandes» obras que se hace posible reconocer la importancia de un lugar o sociedad dentro de los procesos históricos regionales. La importancia de puntos de cruce o «singulares» estaría señalada por el tipo de actividades que se desarrollan allí; es conocido que a los centros de comercio confluyen muchos caminos en una relación que no necesariamente es causal (Menéndez \& Soria, 1994: 79). Los lugares de cruce de caminos, también podrían señalar lugares o centros de importancia económica, cósmica o ritual, y tal importancia o singularidad podría estar marcada por elementos naturales o artificiales fácilmente observables e identificables; pero además, estaría marcada por los toponímicos asociados a ellos, los cuales deberían también ser objeto de estudio.

«lgualmente es necesario asumir que independientemente del tipo de camino que nos encontremos, éstos suplen necesidades específicas, por lo tanto es posible hablar de ellos como estructuras que fueron construidas y usadas correspondiendo a una intencionalidad social e individualmente conciente, y posible en la medida que se tenía un conocimiento profundo de un territorio, el cual se construye y consolida mental y físicamente» (Menéndez \& Soria, 1994: 72).

Desafortunadamente la arqueología y la historia han trabajado por separado y ninguno de los temas aquí mencionados ha sido explorado sistemáticamente. Nuestro punto de partida resulta de la exhaustiva revisión documental realizada por Botero (2005), la cual permitió evidenciar que el accionar español sobre los caminos en Colombia, estuvo determinado por la existencia de la red vial prehispánica, la cual no solo atravesaba y comunicaba todos los rincones y nichos de interés para el establecimiento humano, alcanzando grandes distancias, sino que estaba tan sólidamente construida, que el mayor esfuerzo de los colonizadores se debió concentrar en su destrucción, se debió picar — literalmente- para permitir el paso de mulas y caballos. Nos preguntamos cuál fue la situación en otros lugares del continente. 


\section{Referencias citadas}

ARCINIEGAS, G., 1995 - Caminos reales: caminos del mar, caminos en tierra. In: Caminos reales de Colombia: 19-31; Bogotá: Fondo FEN.

BOTERO PÁEZ, S., 2005 - Caminos ásperos y fragosos para los caballos. Apuntes para la historia de los caminos en Antioquia, 249 p.; Medellín: Comité para el Desarrollo de la Investigación (CODI), Centro de Investigaciones Sociales y Humanas (CISH), Universidad de Antioquia.

CARDALE DE SCHRIMPFF, M., 1996 - Caminos prehispánicos en Calima. El estudio de los caminos precolombinos de la cuenca del alto río Calima, Cordillera Occidental valle del Cauca, 187 p.; Santafe de Bogotá: FIAN, Banco de la República, Fundación Pro Calima.

CARDALE DE SCHRIMPFF, M. \& HERRERA, L., 1995 - Caminos y comerciantes en el suroccidente de Colombia entre 2500 y 1500 A. P. In: Perspectivas regionales en el suroccidente de Colombia y norte del Ecuador (C. Gnecco, ed.): 195-222; Popayán: Universidad del Cauca.

CARDALE DE SCHRIMPFF, M. \& HERRERA, L. (eds.), 2000 - Caminos precolombinos. Las vías, los ingenieros y los viajeros, 300 p.; Bogotá: ICANH, Ministerio de Cultura.

COELLO RODRÍGUEZ, A., 2000 - El camino Inca en el distrito de San Damián (Provincia de Huarochirí, Perú). In: Caminos precolombinos. Las vías, los ingenieros y los viajeros (L. Herrera \& M. Cardale, eds.): 167-193; Bogotá: ICANH, Ministerio de Cultura.

GONZÁLEZ ESCOBAR, L. F., 2001 - Caminos Republicanos en Antioquia. Los caminos de Medellín a Rionegro, las rutas por Santa Elena. www.bibliotecavirtualdeantioquia.gov.co

HAGEN, V. W. von, 1977 - La carretera del sol. La búsqueda del camino real de los incas, 375 p.; México: Editorial Diana.

HYSLOP, J., 1992 - Qhapanan, el sistema vial incaico, 298 p.; Lima: Instituto Andino de Estudios Arqueológicos, Petróleos del Perú.

JIMÉNEZ MENESES, O., PÉREZ MORALES, E. \& GUTIÉRREZ FLÓREZ, F. (eds.), 2005 Caminos, rutas y técnicas: huellas espaciales y estructuras sociales en Antioquia; Medellín: Universidad Nacional de Colombia.

LANGEBAEK, C. H., 1995 - Los caminos aborígenes. Caminos, mercaderes y cacicazgos: circuitos de comunicación antes de la invasión española. In: Caminos reales de Colombia: 35-45; Bogotá: Fondo FEN.

LANGEBAEK, C. H., 2001 - Reseña de caminos precolombinos, las vías, los ingenieros y los viajeros. Arqueología del Área Intermedia, 3: 195-201; Bogotá: Instituto Colombiano de Antropología e Historia, Sociedad Colombiana de Arqueología.

LANGEBAEK, C. H., GIRALDO, S., BERNAL, A., MONROY, S., BARRAGÁN, A. \& MORALES, J., 2000 - Por los caminos del piedemonte. Una historia de las comunicaciones entre los Andes Orientales y los Llanos. Siglos XVI a XIX, 141 p.; Bogotá: Universidad de los Andes. Estudios Antropológicos 2.

MENÉNDEZ, J. R. \& SORIA, A., 1994 - El territorio como artificio cultural. Corografía histórica del norte de la Península Ibérica. Ciudad y territorio. Estudios territoriales, vol. II (99): 63-94; España: Ministerio de Obras Públicas, Transportes y Medio Ambiente. Separata.

OYUELA CAICEDO, A., 1990 - Las redes de caminos prehispánicos en la Sierra Nevada de Santa Marta. In: Ingenierías prehispánicas (S. Mora, ed.): 47-71; Bogotá: ICANH, Fondo FEN. 
PATIÑO, V. M., 1991 - Historia de la cultura material en la América Equinoccial, t. III, 521 p.; Bogotá: Instituto Caro y Cuervo.

REICHEL DOLMATOFF, G., 1997 [1986] - Arqueología de Colombia. Un texto introductorio; Bogotá: Segunda Expedición Botánica, FUNDABOTÁNICA, Biblioteca de la Presidencia de la República de Colombia.

REPIZO CABRERA, C. R., 1994 - La mula. Nueva Revista Colombiana de Folclor, vol. 3 (14): 83-91; Bogotá.

ROBLEDO, J. (atribuido), 1993 [1539-1542] - Relación de Anzerma. In: Relaciones y visitas a los Andes. Siglo XVI (H. Tovar Pinzón, ed.): 335-361; Bogotá: Hermes, Colcultura e Instituto de Cultura Hispánica. Colección de Historia de la Biblioteca Nacional.

SALGADO LÓPEZ, H., 1986 - Asentamientos prehispánicos en el noroccidente del departamento del Valle del Cauca; Bogotá: FIAN, Banco de la República, Talleres Gráficos de Fotolito América.

SAÉNZ SAMPER, J., 2001 - Las águilas doradas: más allá de las fronteras y del tiempo. El motivo de las aves con alas desplegadas en la orfebrería tairona. Boletín Museo del Oro, 48: enero-junio; Bogotá: Banco de la República. http://www.banrep.gov.co/museo/esp/boletin

SZASDI, N. A., 1983 - Corrientes del comercio prehispánico, metales, piedras preciosas y obsidianas, 150 p.; Valladolid: Seminario Americanista de la Universidad Casa de Colón. Cuadernos Prehispánicos 10.

VELANDIA, R., 1996 - Descubrimientos y caminos de los Llanos Orientales, Bogotá: Colcultura. Colección de Historia de la Biblioteca Nacional.

VÉLEZ ESCOBAR, N., 1999 - Caminos antiguos del Medellín sin carreteras. Territorio Cultural. Revista de la Secretaría de Educación y Cultura de Antioquia, 2: 65-70; Antioquia: Dirección de Cultura.

VÉLEZ, N. \& BOTERO, S., 1997 - La Búsqueda del Valle de Arví y descubrimiento de los valles de Aburrá y Rionegro por el Capitán Jorge Robledo, 140 p.; Medellín: Comisión Asesora para la Cultura del Concejo de Medellín. 